\title{
Incident diabetes mellitus may explain the association between sleep duration and incident coronary heart disease
}

\author{
Akiko Kishi Svensson ${ }^{1,2,3,4}$ - Thomas Svensson ${ }^{1,4,5}$ (1) Mariusz Kitlinski ${ }^{1,6}$. \\ Peter Almgren $^{1}$ • Gunnar Engström ${ }^{1}$ • Peter M. Nilsson ${ }^{1}$ • Olle Melander ${ }^{1,7}$
}

Received: 10 June 2017 / Accepted: 6 September 2017 / Published online: 4 November 2017

(C) The Author(s) 2017. This article is an open access publication

\begin{abstract}
Aims/hypothesis Sleep duration is a risk factor for incident diabetes mellitus and CHD. The primary aim of the present study was to investigate, in sex-specific analyses, the role of incident diabetes as the possible biological mechanism for the reported association between short/long sleep duration and incident CHD. Considering that diabetes is a major risk factor for CHD, we hypothesised that any association with sleep duration would not hold for cases of incident CHD occurring before incident diabetes ('non-diabetes CHD') but would hold true for cases of incident CHD following incident diabetes ('diabetes-CHD').

Methods A total of 6966 men and 9378 women aged 4573 years from the Malmö Diet Cancer Study, a populationbased, prospective cohort, who had answered questions on habitual sleep duration and did not have a history of prevalent diabetes or CHD were included in the analyses. Incident cases of diabetes and CHD were identified using national registers. Sex-specific Cox proportional hazards regression models
\end{abstract}

Akiko Kishi Svensson and Thomas Svensson contributed equally to this study.

Electronic supplementary material The online version of this article (https://doi.org/10.1007/s00125-017-4464-3) contains peer-reviewed but unedited supplementary material, which is available to authorised users.

Thomas Svensson

thomas.svensson@med.lu.se

1 Department of Clinical Sciences, Lund University, Skåne University Hospital, CRC, Jan Waldenströms gata 35, 20502 Malmö, Sweden

2 Department of Diabetes and Metabolic Diseases, University of Tokyo, Tokyo, Japan

3 Clinical Research Support Center, University of Tokyo Hospital, Tokyo, Japan were stratified by BMI and adjusted for known covariates of diabetes and $\mathrm{CHD}$.

Results Mean follow-up times for incident diabetes $(n=1137 /$ 1016 [men/women]), incident CHD $(n=1170 / 578)$, nondiabetes CHD $(n=1016 / 501)$ and diabetes-CHD $(n=154 /$ 77) were 14.2-15.2 years for men, and 15.8-16.5 years for women. In men, short sleep duration $(<6 \mathrm{~h})$ was associated with incident diabetes (HR 1.35, 95\% CI 1.01, 1.80), CHD (HR 1.41, 95\% CI 1.06, 1.89) and diabetes-CHD (HR 2.34, 95\% CI 1.20, 4.55). Short sleep duration was not associated with incident non-diabetes CHD (HR 1.35, 95\% CI 0.98, 1.87). Long sleep duration ( $\geq 9 \mathrm{~h}$ ) was associated with incident diabetes (HR 1.37, 95\% CI 1.03, 1.83), CHD (HR 1.33, 95\% CI 1.01, 1.75) and diabetes-CHD (HR 2.10, 95\% CI $1.11,4.00)$. Long sleep duration was not associated with incident non-diabetes CHD (HR 1.33, 95\% CI 0.98, 1.80). In women, short sleep duration was associated with incident diabetes (HR 1.53, 95\% CI 1.16, 2.01), CHD (HR 1.46, 95\% CI $1.03,2.07$ ) and diabetes-CHD (HR 2.88, 95\% CI 1.37, 6.08).

4 Center of Innovation, University of Tokyo, Tokyo, Japan

5 Department of Neuropsychiatry, Keio University School of Medicine, Tokyo, Japan

6 Department of Cardiology, Skåne University Hospital, Malmö, Sweden

7 Department of Internal Medicine, Skåne University Hospital, Malmö, Sweden 
Short sleep duration was not associated with incident nondiabetes CHD (HR 1.29, 95\% CI 0.86, 1.93).

Conclusions/interpretation The associations between sleep duration and incident CHD directly reflect the associations between sleep duration and incident diabetes. Incident diabetes may thus be the explanatory mechanism for the association between short and long sleep duration and incident CHD.

Keywords Cohort $\cdot$ Coronary heart disease $\cdot$ Diabetes mellitus $\cdot$ Epidemiology $\cdot$ Incidence $\cdot$ Sleep duration

\author{
Abbreviations \\ CABG Coronary artery bypass graft \\ MDC Malmö Diet and Cancer \\ PCI Percutaneous coronary intervention
}

\section{Introduction}

A suggested novel risk factor for incident diabetes mellitus is sleep duration; insufficient or excessive amounts of sleep may contribute to the development of diabetes, with prospective studies reporting that short but not long sleep duration [1-4], or both short and long sleep duration, are associated with incident diabetes [5-7]. Meta-analyses support a U-shaped association between sleep duration and incident type 2 diabetes $[8,9]$. Furthermore, sleep duration predicts cardiovascular outcomes [10], and both short [11-14] and long [14, 15] sleep time is associated with incident CHD in prospective studies. The importance of sleep for cardiovascular health has prompted the American Heart Association to recently release a scientific statement outlining clinical recommendations and future research directions on sleep behaviour [16].

Any positive association between excessive or insufficient sleep duration and incident CHD must, however, be considered in the context of diabetes as a major risk factor for CHD [17]. Cardiometabolic risk factors, including prevalent type 2 diabetes, have been discussed as possible mediators for the association between sleep duration and CHD [11, 14], with prospective studies adjusting for prevalent baseline cases of diabetes in their analyses [11-15]. This may be insufficient as the same studies do not take into account the incident cases of diabetes occurring during follow-up. Incident diabetes may represent a change in the risk of developing future CHD, yet no study to date has been able to conclusively demonstrate or refute whether only those who are diagnosed with incident diabetes during follow-up constitute a specific group in which short and long sleep duration are associated with incident CHD.

Sex-stratified analyses are already an established method in cardiovascular research. However, there is emerging evidence of the importance of sex-stratification in research involving diabetes-related risk factors $[18,19]$ and outcomes $[17,20]$.
Sex-stratified analyses are thus called for to identify sexspecific diabetes and CHD risk factors and their mechanisms [17]. Sleep duration may be one such important sex-specific lifestyle-related risk factor [21].

The primary objective of the present study was thus to investigate, in sex-specific analyses, the role of incident diabetes as the possible biological mechanism for the reported association between short/long sleep duration and incident CHD. Such a crucial role of incident diabetes can be confirmed in four steps: (1) if an association between sleep duration and incident diabetes is established, and (2) an association between sleep duration and incident CHD is confirmed, it is possible to investigate whether the latter association persists when considering (3) only cases of incident CHD occurring prior to incident diabetes, and (4) only cases of incident CHD occurring after incident diabetes, respectively.

We hypothesised that any association between sleep duration and incident CHD would be significant only in individuals who, during follow-up, were diagnosed with diabetes before CHD. In accordance with this hypothesis, we expected no association between sleep duration and incident CHD occurring before incident diabetes, but an increased risk of incident CHD occurring after incident diabetes. In order to address the possibility of sex-specific risks, all analyses were conducted separately for men and women.

\section{Methods}

The Malmö Diet and Cancer (MDC) Study is a populationbased, prospective study run in the city of Malmö, Sweden, whose details have been described elsewhere [22]. In brief, men and women between the ages of 45 and 73 years were randomly selected and recruited for a baseline examination between the years 1991 and 1996. Participants' anthropometric data and blood samples were gathered, in addition to responses to a detailed questionnaire on heredity, socioeconomic variables, social network, occupation, physical activity, alcohol consumption, smoking, diseases and medication.

At baseline, 30,447 individuals were identified in the study population (see electronic supplementary material [ESM] Fig. 1). Participants of the present study were excluded if they had a history of prevalent diabetes $(n=1340)$ or CHD ( $n=670$ ), provided incomplete information on sleep duration ( $n=12,048)$ or provided a sleep duration that represented an outlier value of more than three interquartile ranges below or above the first and fourth quartiles respectively $(n=45)$. A total of 6966 men and 9378 women were included in the analyses. Participants were followed from the starting point until 31 December 2010, with person-years calculated from the starting point to the date of the incident event, loss to follow-up or the end of the follow-up period, whichever came first. 
The MDC study was approved by the ethics committee at Lund University, and all participants provided written informed consent.

Sleep duration Habitual sleep duration was assessed through two open questions asking participants how long they slept on weekdays and weekends, respectively. The two questions were formulated as: (a) 'How many hours do you usually sleep per night during a typical week (Monday-Friday)?' and (b) 'How many hours do you usually sleep per night during a typical weekend (Saturday-Sunday)?' A weighted average sleep duration [(weekday $\times 5)+($ weekend $\times 2) / 7]$ was calculated for individuals who responded to both questions ( $n=16,344)$, thereby allowing the subsequent construction of a categorical variable consisting of five sleep duration groups ( $<6 \mathrm{~h}, 6-7 \mathrm{~h}, 7-8 \mathrm{~h}, 8-9 \mathrm{~h}$ and $\geq 9 \mathrm{~h}$ ). Because only $0.9 \%$ ( $n=146)$ of the population slept for $<5 \mathrm{~h}$, short and long sleep duration were defined as $<6 \mathrm{~h}$ and $\geq 9 \mathrm{~h}$, respectively. The reference category ( $7-8 \mathrm{~h}$ ) was chosen on the basis of the lowest incidence rates of all endpoints in this particular group.

Incident diabetes mellitus Incident diabetes was defined as new-onset diabetes in individuals without prevalent diabetes at baseline. Prevalent diabetes at the MDC baseline examination was defined as any of the following: having a measured fasting blood glucose $\geq 6.1 \mathrm{mmol} / 1$ (corresponding to fasting plasma glucose concentration $\geq 7 \mathrm{mmol} / \mathrm{l}$ ) at the MDC baseline examination, or a self-reported history of physiciandiagnosed diabetes, or use of diabetes medication according to the MDC baseline questionnaire, or being diagnosed and registered in any of the local or national diabetes registries as previously described [23].

The process of endpoint retrieval has been described in detail elsewhere [24]. In brief, individuals with incident diabetes were identified through linkage of a 10-digit national personal identification number with six local and national registers: the Malmö $\mathrm{HbA}_{1 \mathrm{c}}$ register, the Regional Diabetes 2000 register of the Scania region [25], the Swedish National Diabetes Register [26], the Swedish National Inpatient Register [27], the Swedish Cause of Death Register [28] and the Swedish Prescribed Drug Register [29]. Apart from being captured in these registers, individuals with incident diabetes could also be captured by having a fasting plasma glucose concentration $\geq 7 \mathrm{mmol} / 1$ or a $120 \mathrm{~min}$ plasma glucose value of $>11.0 \mathrm{mmol} / \mathrm{l}$ in subpopulations of the MDC participating in a MDC re-examination [30] or the Malmö Preventive Project re-examination. [31]

Incident coronary heart disease Time to first occurrence of a CHD event was defined as a first fatal or non-fatal myocardial infarction, coronary artery bypass graft (CABG) or percutaneous coronary intervention (PCI). All events were identified through linkage of a 10-digit national personal identification number with three registries validated for classification of outcomes as described elsewhere [32, 33]: the Swedish National Discharge Registry, the Swedish Cause of Death Register, and the Swedish Coronary Angiography and Angioplasty Registry. CABG and PCI were classified using the national classification of surgical procedures operation codes (KKÅ or Op6): 3065, 3066, 3068, 3080, 3092, 3105, 3127, 3158 for CABG, and FNG02 and FNG05 for PCI. A coronary event was defined according to the ICD-9 (www.icd9data.com/ 2007/Volume1) and ICD-10 (www.who.int/classifications/ $\mathrm{icd} / \mathrm{en} /$ ) revisions with fatal or non-fatal myocardial infarction or death due to CHD corresponding to codes 410, 412, and 414 (ICD-9), and I21-I23 and, I25 (ICD-10).

Incident CHD in relation to incident diabetes In order to elucidate the role of diabetes as the explanatory mechanism for the association between sleep duration and CHD, two additional endpoint variables were created: (1) 'non-diabetes CHD', defined as an incident CHD event occurring in participants without incident diabetes, or in those in whom incident diabetes occurred after incident CHD; and (2) 'diabetesCHD', defined as incident CHD diagnosed on the same day, or following a diagnosis of incident diabetes.

Statistical analyses Sex-specific risks for the association between sleep duration and incident diabetes were estimated using Cox proportional hazards regression. The minimally adjusted models were adjusted for age (continuous) and socioeconomic index at starting point. Multivariable models were additionally adjusted for marital status (married, single, divorced or widowed), smoking (never, past and current smoker: $<20$ cigarettes/day, or $\geq 20$ cigarettes/day), alcohol intake (none, $0.1-1.6,1.7-7.2,7.3-15.4$ or $\geq 15.5 \mathrm{~g}$ ethanol/day), physical activity, hypertension, use of lipid-lowering medication, shift work (yes/no), psychological stress (low, intermediate and high) and sleep quality. Missing data were addressed through the construction of dummy variables. All multivariable models were stratified by four categories of BMI $\left(<18.5 \mathrm{~kg} / \mathrm{m}^{2}, 18.5-\right.$ $24.9 \mathrm{~kg} / \mathrm{m}^{2}, 25-29.9 \mathrm{~kg} / \mathrm{m}^{2}$ and $\geq 30 \mathrm{~kg} / \mathrm{m}^{2}$ ), using the Stata option 'strata()' to account for different baseline hazards across BMI categories.

Socioeconomic index was categorised according to the Swedish socioeconomic classification [34] (manual worker, low and intermediate level non-manual worker, higher level non-manual worker, other [self-employed including farmers] and unemployed). Physical activity was defined as leisure time physical activity based on 18 items adapted from the Minnesota Leisure Time Physical Activity instrument and has been described in detail elsewhere [35]. Hypertension was defined as systolic blood pressure $\geq 140 \mathrm{mmHg}$, diastolic blood pressure $\geq 90 \mathrm{mmHg}$ or the use of antihypertensive medication (angiotensin-converting enzyme inhibitors, $\beta$-blockers, calcium antagonists or diuretics). Psychological 
stress was constructed from validated questions assessing psychosocial work characteristics measuring job strain, and from one question assessing non-occupational stress. Sleep quality was determined through four questions assessing (1) difficulty initiating sleep, (2) difficulty maintaining sleep, (3) early morning awakening, and (4) not feeling rested.

Sensitivity analyses excluded the first 3 years of follow-up to minimise the chance of reverse causation.

All statistical analyses were performed using Stata version 13.1 SE (StataCorp LP, College Station, TX, USA). $p$ values were two-tailed and considered significant if $p<0.05$.

\section{Results}

Compared with those who did not provide information on sleep duration, the group that was included in all analyses had a higher proportion of men (42.6\% vs $32.4 \%)$. Moreover, compared with those who were included (mean age and SD for both men and women, $57.3 \pm 6.0$ years), men who did not provide information on sleep duration were older (61.3 \pm 7.8 years), whereas women were slightly younger (57.0 \pm 9.6 years).

Mean sleep duration for men and women was $7.3 \mathrm{~h}$ $(\mathrm{SD} \pm 0.9 \mathrm{~h}$ ). Table 1 summarises baseline characteristics stratified according to sex. The largest proportion of men $(42.9 \%)$ and women $(40.9 \%)$ had a sleep duration of 7-8 h. Compared with those with short $(<6 \mathrm{~h})$ and long $(\geq 9 \mathrm{~h})$ sleep durations, men and women in the reference category (7-8 h) were less likely to be obese, do high levels of physical activity or shift work, and smoke more than 20 cigarettes/day, and more likely to be married and report low levels of psychological stress. Men who reported 7-8 h of sleep were also less likely to be hypertensive and less likely to use lipid-lowering medication.

Incident diabetes Mean follow-up time for analysis of incident diabetes was 15.0 years for men and 16.0 years for women. During follow-up, $16.3 \%$ of men $(n=1137)$ and $10.8 \%$ of women $(n=1016)$ developed diabetes.

In the multivariable analysis for men, short sleep duration was borderline significantly associated with incident diabetes (HR $1.26,95 \%$ CI $0.96,1.65$ ), while long sleep duration was significantly associated with an increased risk of incident diabetes (HR 1.31, 95\% CI 1.00, 1.71) compared with the reference category (Table 2). When excluding the first 3 years of follow-up, men with short (HR 1.35, 95\% CI 1.01, 1.80) and long (HR 1.37, 95\% CI 1.03, 1.83) sleep durations had an increased risk of incident diabetes.

Among women, short sleep duration was associated with incident diabetes in the fully adjusted model (HR 1.55, 95\% CI 1.20, 2.01) and when excluding the first 3 years of followup (HR 1.53, 95\% CI 1.16, 2.01) (Table 2). Long sleep duration was not associated with incident diabetes in women.
Incident CHD Mean follow-up time for analysis of incident CHD was 15.2 years for men and 16.5 years for women. During follow-up, $16.8 \%$ of men $(n=1170)$ and $6.2 \%$ of women $(n=578)$ developed CHD.

In the fully adjusted models for men, short sleep duration was associated with incident CHD (HR 1.32, 95\% CI 1.01, 1.73) (Table 3 ). When excluding the first 3 years of follow-up, both short (HR 1.41, 95\% CI 1.06, 1.89) and long (HR 1.33, $95 \%$ CI $1.01,1.75$ ) sleep duration were associated with an increased risk of CHD.

For women, short sleep duration was associated with an increased risk of CHD in the fully adjusted model (HR 1.41, $95 \%$ CI $1.00,1.99$ ), and in the sensitivity analysis (HR 1.46, $95 \%$ CI 1.03, 2.07) (Table 3). Long sleep duration was not associated with any increase in risk of CHD.

Incident non-diabetes CHD Mean follow-up time for analysis of incident non-diabetes CHD was 14.2 years for men and 15.8 years for women. During follow-up, $14.6 \%$ of men $(n=1016)$ and $5.3 \%$ of women $(n=501)$ developed nondiabetes CHD.

In the multivariable model for men, neither short (HR 1.23, $95 \%$ CI $0.91,1.66$ ) nor long (HR $1.13,95 \%$ CI $0.84,1.50$ ) sleep duration were associated with an increased risk of incident non-diabetes CHD (ESM Table 1). When excluding the first 3 years of follow-up, the results for short (HR 1.35, 95\% CI $0.98,1.87$ ) and long (HR 1.33, 95\% CI 0.98, 1.80) sleep duration remained non-significantly associated with incident non-diabetes CHD.

In the multivariable model for women, there was no significant association between short (HR 1.24, 95\% CI 0.83, 1.84) or long (HR 1.28, 95\% CI $0.87,1.88$ ) sleep duration and incident non-diabetes CHD (ESM Table 1). When excluding the first 3 years of follow-up, the results for short (HR 1.29, $95 \%$ CI $0.86,1.93$ ) and long (HR $1.34,95 \%$ CI $0.90,1.98$ ) sleep duration remained non-significantly associated with incident non-diabetes CHD.

Incident diabetes-CHD Mean follow-up time for analysis of incident diabetes-CHD was 14.4 years for men and 15.9 years for women. During follow-up, $2.2 \%$ of men $(n=154)$ and $0.8 \%$ of women $(n=77)$ developed diabetes-CHD.

In the multivariable model for men, short (HR 2.37, 95\% CI 1.22, 4.60) and long (HR 2.21, 95\% CI 1.16, 4.19) sleep duration was associated with an increased risk of incident diabetes-CHD (Table 4). When excluding the first 3 years of follow-up, both short (HR 2.34, 95\% CI 1.20, 4.55) and long (HR 2.10, 95\% CI 1.11, 4.00) sleep duration were associated with an increased risk of incident diabetes-CHD.

In women, short sleep duration was associated with an increased risk of diabetes-CHD in both the multivariable model (HR $2.93,95 \%$ CI $1.39,6.16)$ and the sensitivity analysis (HR 2.88, 95\% CI 1.37, 6.08) (Table 4). 


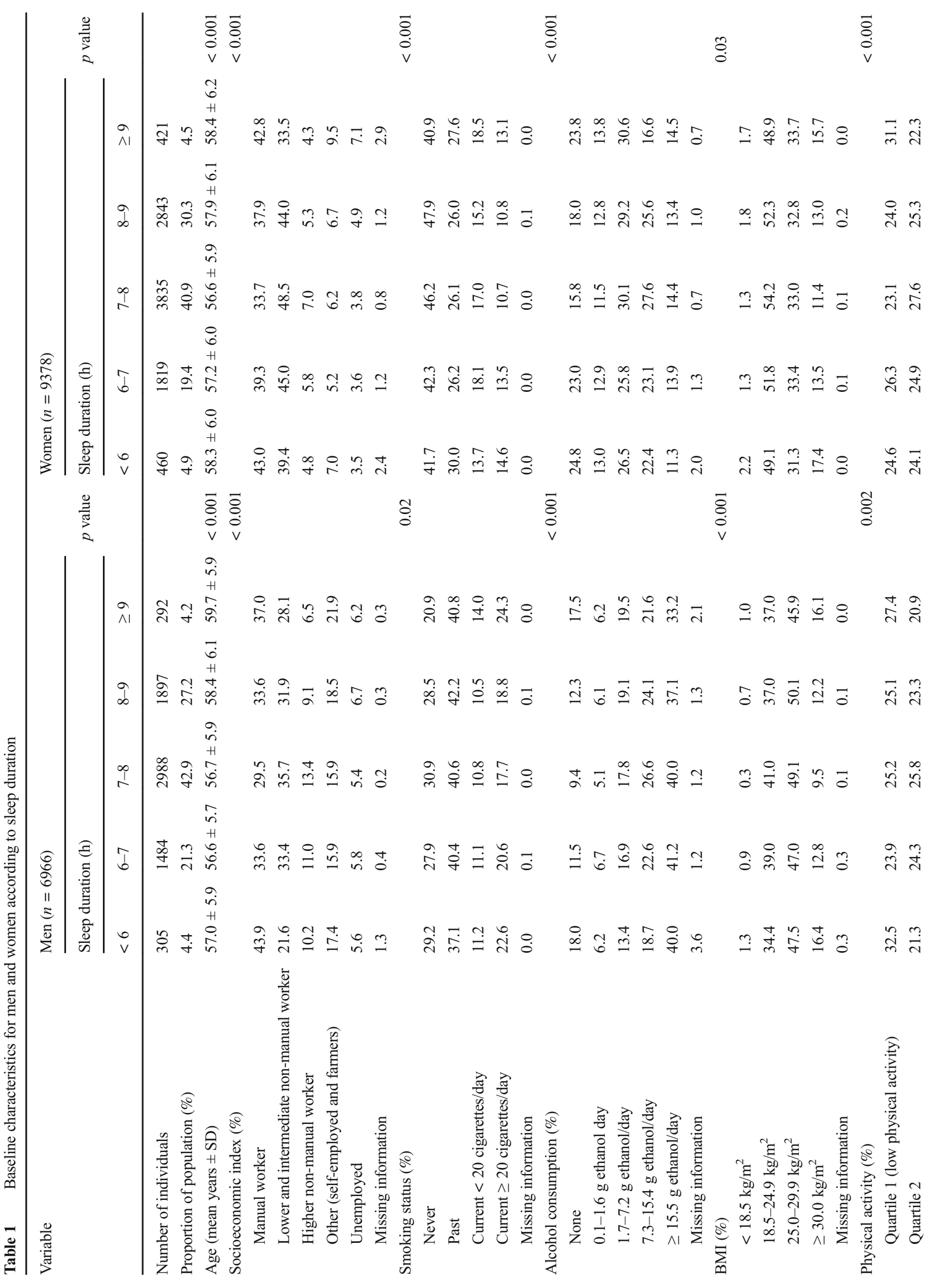




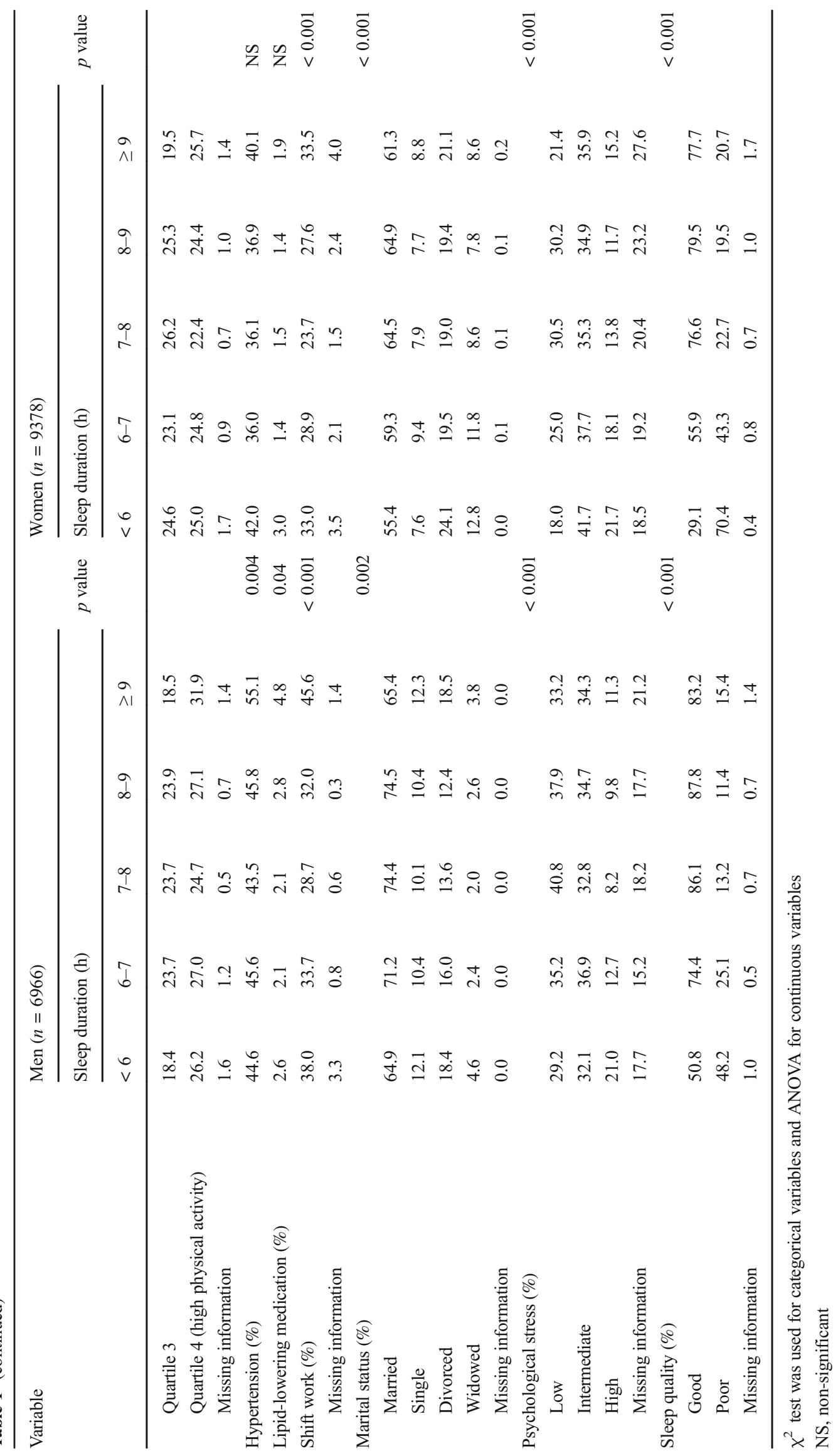




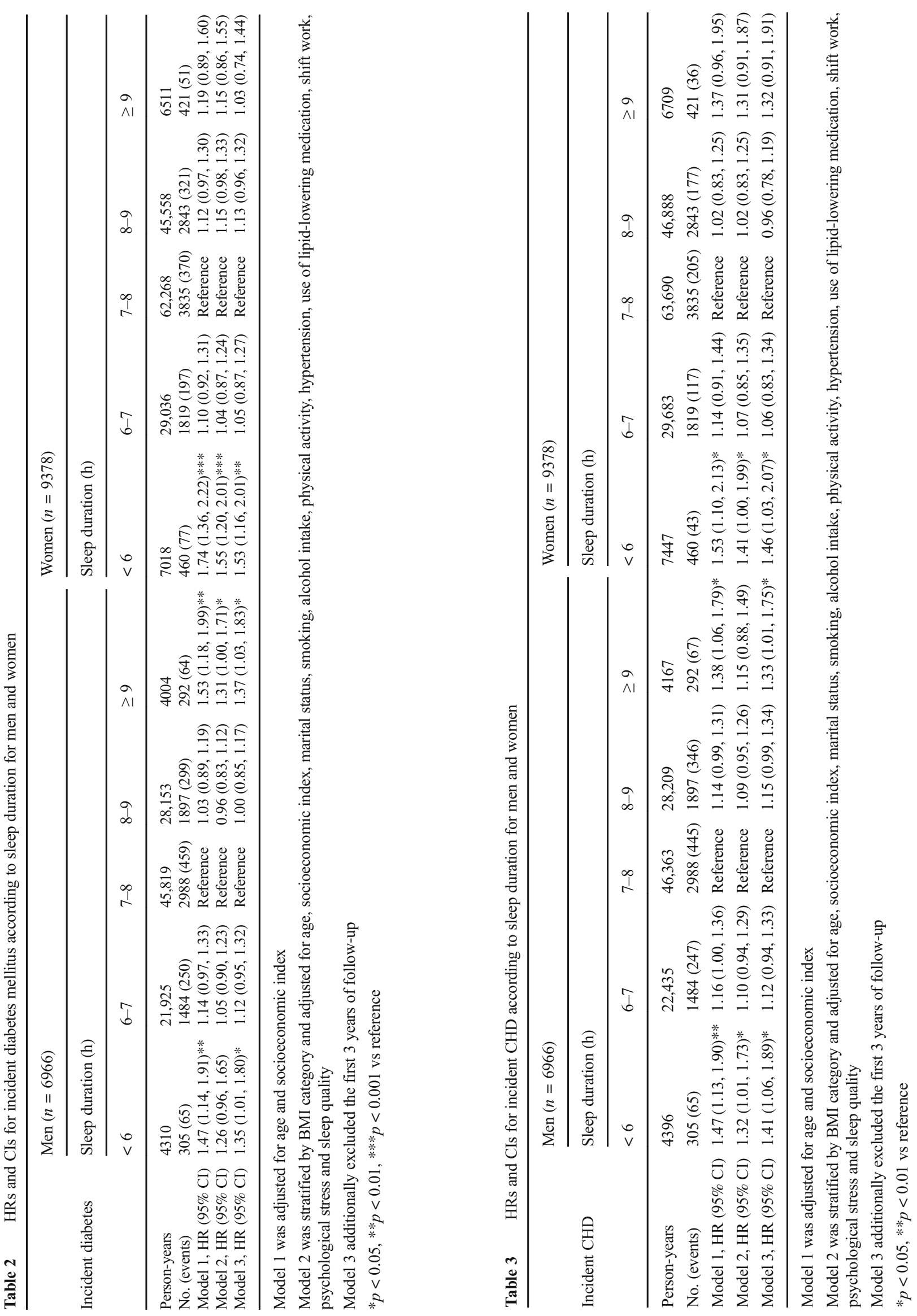




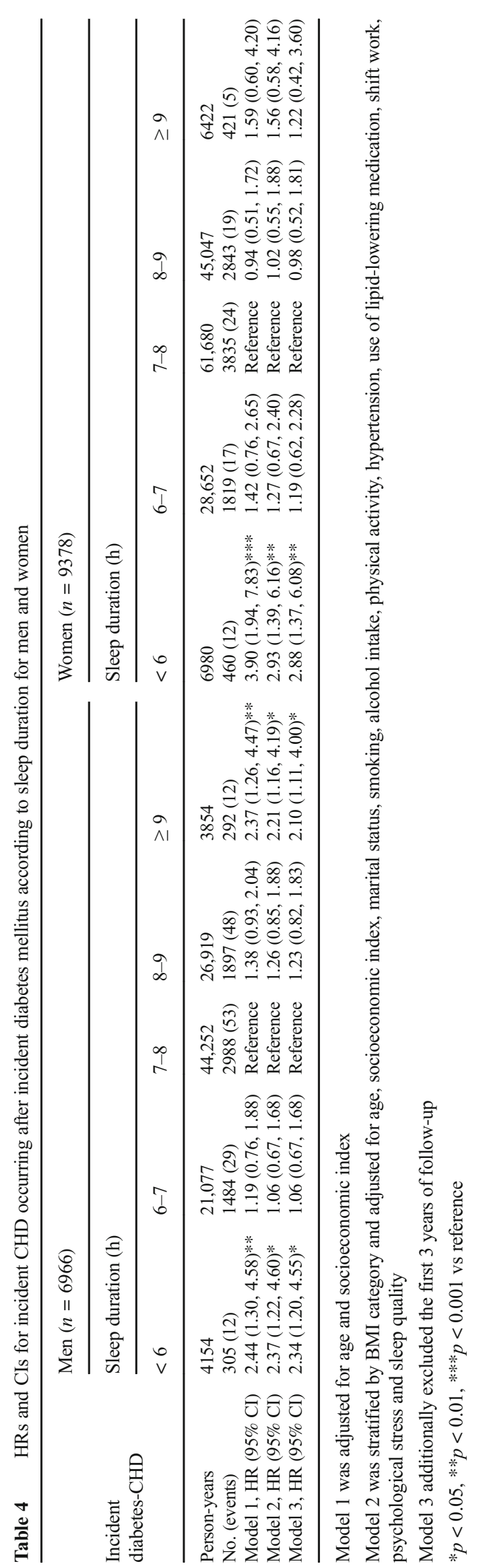




\section{Discussion}

Our study is the first of its kind to consider incident diabetes on the pathway between self-reported sleep duration and CHD risk. The associations between sleep duration and incident CHD directly reflect the associations between sleep duration and incident diabetes, and when taken together with the strong association between sleep duration and diabetes-CHD, our study convincingly demonstrates that incident diabetes is the most probable explanatory biological mechanism for the positive associations found between sleep duration and CHD.

The association between short sleep duration and incident diabetes in our study is consistent with previous prospective findings [1-7]. Although both BMI and hypertension have been mentioned as possible mediators [6] for the association between short sleep duration and incident diabetes, the association between short sleep duration and incident diabetes in our study persisted in both men and women despite BMIstratified analyses and adjustment for a large number of known diabetes risk factors and covariates, including hypertension. Prospective studies with repeated measurements of BMI and blood pressure are, however, urged to investigate the importance of any change in these variables over time for the association between sleep duration, incident diabetes and incident CHD, respectively.

The increased risk of CHD with short sleep duration [11-14] is also consistent with previous prospective studies and could easily have been argued as an increased risk independent of diabetes considering the exclusion of individuals with prevalent diabetes from our analyses. However, we chose to take into account the association between habitually short sleep duration and incident diabetes and opted for an approach that (1) censored cases of incident CHD if these occurred after incident diabetes (non-diabetes CHD), thereby obliterating any significant association between short sleep duration and CHD; and (2) considered only cases of incident CHD that followed incident diabetes (diabetes-CHD), which in turn showed significant positive associations between short sleep duration and CHD despite the low number of cases. This novel approach recognises the important role of incident diabetes as the explanatory mechanism for any association between short sleep duration and incident CHD in both men and women.

Our study also found that long sleep duration $(\geq 9 \mathrm{~h})$ in men is associated with incident diabetes and diabetes-CHD, but not with non-diabetes CHD. Contrary to the situation with short sleep duration, the associations between long sleep duration and incident diabetes and CHD, respectively, have been suggested to result from comorbidity and residual confounding $[5,10]$ or from reverse causation bias $[2,14]$. In order to address the problem of reverse causation, our study excluded individuals with prevalent diabetes and CHD, and allowed for sensitivity analyses that further excluded the first 3 years of follow-up. Despite this approach, long sleep duration in men remained significantly positively associated with incident diabetes, incident $\mathrm{CHD}$ and incident diabetes-CHD, but not with incident non-diabetes CHD. The main reason for a null finding between long sleep duration and incident CHD in women is most likely due to the lack of any association between long sleep duration and incident diabetes in this group, a finding consistent with one previous report [1]. We therefore propose for the first time that incident diabetes is also the responsible mechanism for the observed association between long sleep duration and incident CHD in men.

The findings are strong and point toward diabetes as the explanatory biological mechanism for the association between sleep duration and CHD, and the fact that this association may differ between men and women. The significance of this finding is threefold. First, it highlights that sleep duration should be considered an important behavioural risk factor for incident diabetes. Second, the relevance of our findings is not limited to $\mathrm{CHD}$, owing to the importance of diabetes as a major risk factor for micro- and macrovascular disease. Sleep interventions in individuals who are habitually short or long sleepers thus have the potential to greatly impact health outcomes and should be considered on a par with advice on physical exercise. Indeed, the American Heart Association has recently published a scientific statement [16] outlining clinical recommendations and research priorities on sleep behaviour owing to the strong association between sleep and cardiovascular health. Third, the present results strengthen the need for sexstratified analyses with regard to diabetes risk factors and CHD complications. The main sex difference that emerges from our study is that long sleep duration is a risk factor for incident diabetes and incident diabetes-CHD in men but not women. Such differences between men and women may be due to underlying biological as well as psychosocial influences [36], which may determine sex-specific diabetes risk factors [18]. Indeed, sex differences in the association between sleep duration and incident diabetes have already been reported [1], and the impact of sleep duration on body composition may differ according to sex [21]. Mechanistic studies are required to explain the sex-specific effects of sleep duration and their association with incident diabetes and incident CHD, respectively. Future research on this topic is thus highly warranted.

There are a few limitations to this study. First, sleep was assessed through self-reported questionnaires, with possible overestimation of sleep duration. However, any nondifferential misclassification of sleep duration among our participants would consequently result in a subsequent underestimation of our study findings. Additionally, when considering the nature and size of our study, no other feasible methods of assessing sleep duration exist. Second, the lack of an association between long sleep duration and the diabetes-CHD endpoint in women could be due to a lack of statistical power because of the small number of individuals involved. 
Studies with a larger population and longer follow-up times are encouraged to conduct sex-stratified analyses and attempt to replicate our results. Third, the MDC study does not contain information on mental illness, and we were therefore unable to adjust for this in our analyses. Fourth, the group of individuals included in analyses differed in age and sex from those who had not provided information on sleep duration. Those included for analysis included a higher proportion of men, and they were also younger than the excluded men. Conversely, the women included were slightly older than their excluded counterparts. This may have biased our findings. Fifth, the generalisability of results to other populations may be limited. Ethnic differences in the association between sleep duration and incident diabetes have been shown in previous research [2], and different ethnicities may require different amounts of sleep before such behaviours constitute a health hazard.

Despite such limitations, this study has a number of strengths. First, incident diabetes and incident CHD were established using nationwide registers, with very high accuracy. Second, the MDC questionnaire asks about habitual sleep duration, which would be the question of choice if sleep duration were assessed by healthcare professionals. Third, we have used a population that is highly representative of the Swedish general population, and we adjusted for a large number of covariates associated with incident diabetes and CHD. Finally, we adjusted for sleep quality as poor sleep quality may confound any association between short or long sleep duration and health outcomes. With the exception of one prospective study [3], sleep quality has not been considered when positive associations have been reported between sleep duration and incident diabetes.

Conclusion The results of this population-based study demonstrate for the first time that the association between sleep duration and incident CHD may be explained by incident diabetes. Interventions targeting sleep duration could thus have a significant impact on health outcomes when considering the importance of diabetes for a wide range of diseases.

Data availability Owing to ethical and legal restrictions related to the Swedish Biobanks in Medical Care Act (2002:297) and the Personal Data Act (1998:204), data are available upon request from the data access group of MDC Study by contacting Anders Dahlin (anders.dahlin@med.lu.se).

Funding This work was supported by the European Research Council (StG-282,255), the Swedish Heart and Lung Foundation, Swedish Research Council; the Novo Nordisk Foundation, the Skåne University Hospital donation funds; the Medical Faculty, Lund University; the Governmental funding of clinical research within the national health services, the Albert Påhlsson Research Foundation, Region Skåne, the King Gustav V and Queen Victoria Foundation and the Marianne and Marcus Wallenberg Foundation. The funders had no role in study design, in the collection, analysis and interpretation of data, in writing of the article or in the decision to submit the article.
Duality of interest All authors have completed the ICMJE uniform disclosure form at www.icmje.org/coi_disclosure.pdf and declare: no support from any organisation for the submitted work; no financial relationships with any organisations that might have an interest in the submitted work in the previous three years; no other relationships or activities that could appear to have influenced the submitted work.

Contribution statement $\mathrm{AKS}$, TS and $\mathrm{OM}$ were responsible for the conception and design of the study. AKS, TS, OM, MK, PA, GE and PN analysed and interpreted the data. AKS and TS drafted the manuscript. OM, MK, PA, GE and PN critically revised the manuscript for important intellectual content. All authors approved the final version of the manuscript. TS and OM are the guarantors of this work and, as such, had full access to all the data in the study and take responsibility for the integrity of the data and the accuracy of the data analysis. TS had final responsibility for the decision to submit for publication.

Open Access This article is distributed under the terms of the Creative Commons Attribution 4.0 International License (http:// creativecommons.org/licenses/by/4.0/), which permits unrestricted use, distribution, and reproduction in any medium, provided you give appropriate credit to the original author(s) and the source, provide a link to the Creative Commons license, and indicate if changes were made.

\section{References}

1. Mallon L, Broman JE, Hetta J (2005) High incidence of diabetes in men with sleep complaints or short sleep duration: a 12-year followup study of a middle-aged population. Diabetes Care 28:2762-2767

2. Beihl DA, Liese AD, Haffner SM (2009) Sleep duration as a risk factor for incident type 2 diabetes in a multiethnic cohort. Ann Epidemiol 19:351-357

3. Kita T, Yoshioka E, Satoh H et al (2012) Short sleep duration and poor sleep quality increase the risk of diabetes in Japanese workers with no family history of diabetes. Diabetes Care 35:313-318

4. Holliday EG, Magee CA, Kritharides L, Banks E, Attia J (2013) Short sleep duration is associated with risk of future diabetes but not cardiovascular disease: a prospective study and meta-analysis. PLoS One 8:e82305

5. Yaggi HK, Araujo AB, McKinlay JB (2006) Sleep duration as a risk factor for the development of type 2 diabetes. Diabetes Care 29: 657-661

6. Gangwisch JE, Heymsfield SB, Boden-Albala B et al (2007) Sleep duration as a risk factor for diabetes incidence in a large U.S. sample. Sleep 30:1667-1673

7. Chaput JP, Despres JP, Bouchard C, Astrup A, Tremblay A (2009) Sleep duration as a risk factor for the development of type 2 diabetes or impaired glucose tolerance: analyses of the Quebec Family Study. Sleep Med 10:919-924

8. Cappuccio FP, D'Elia L, Strazzullo P, Miller MA (2010) Quantity and quality of sleep and incidence of type 2 diabetes: a systematic review and meta-analysis. Diabetes Care 33:414-420

9. Shan Z, Ma H, Xie M et al (2015) Sleep duration and risk of type 2 diabetes: a meta-analysis of prospective studies. Diabetes Care 38 : 529-537

10. Cappuccio FP, Cooper D, D'Elia L, Strazzullo P, Miller MA (2011) Sleep duration predicts cardiovascular outcomes: a systematic review and meta-analysis of prospective studies. Eur Heart J 32: 1484-1492

11. Hoevenaar-Blom MP, Spijkerman AM, Kromhout D, van den Berg JF, Verschuren WM (2011) Sleep duration and sleep quality in relation to 12-year cardiovascular disease incidence: the MORGEN study. Sleep 34:1487-1492 
12. Meisinger C, Heier M, Lowel H, Schneider A, Doring A (2007) Sleep duration and sleep complaints and risk of myocardial infarction in middle-aged men and women from the general population: the MONICA/KORA Augsburg cohort study. Sleep 30:1121-1127

13. Liu J, Yuen J, Kang S (2014) Sleep duration, C-reactive protein and risk of incident coronary heart disease-results from the Framingham Offspring Study. Nutr Metab Cardiovasc Dis 24: 600-605

14. Ayas NT, White DP, Manson JE et al (2003) A prospective study of sleep duration and coronary heart disease in women. Arch Intern Med 163:205-209

15. Westerlund A, Bellocco R, Sundstrom J, Adami HO, Akerstedt T, Trolle Lagerros Y (2013) Sleep characteristics and cardiovascular events in a large Swedish cohort. Eur J Epidemiol 28:463-473

16. St-Onge MP, Grandner MA, Brown D et al (2016) Sleep duration and quality: impact on lifestyle behaviors and cardiometabolic health: a scientific statement from the American Heart Association. Circulation 134:e367-e386

17. Peters SA, Huxley RR, Woodward M (2014) Diabetes as risk factor for incident coronary heart disease in women compared with men: a systematic review and meta-analysis of 64 cohorts including 858 , 507 individuals and 28,203 coronary events. Diabetologia 57: 1542-1551

18. Logue J, Walker JJ, Colhoun HM et al (2011) Do men develop type 2 diabetes at lower body mass indices than women? Diabetologia 54:3003-3006

19. Wannamethee SG, Papacosta O, Lawlor DA et al (2012) Do women exhibit greater differences in established and novel risk factors between diabetes and non-diabetes than men? The British Regional Heart Study and British Women's Heart Health Study. Diabetologia 55:80-87

20. Roche MM, Wang PP (2013) Sex differences in all-cause and cardiovascular mortality, hospitalization for individuals with and without diabetes, and patients with diabetes diagnosed early and late. Diabetes Care 36:2582-2590

21. St-Onge MP, Perumean-Chaney S, Desmond R et al (2010) Gender differences in the association between sleep duration and body composition: the Cardia Study. Int J Endocrinol 2010:726071

22. Berglund G, Elmstahl S, Janzon L, Larsson SA (1993) The Malmo Diet and Cancer Study. Design and feasibility. J Intern Med 233: 45-51

23. Enhorning S, Sjogren M, Hedblad B, Nilsson PM, Struck J, Melander O (2016) Genetic vasopressin 1b receptor variance in overweight and diabetes mellitus. Eur J Endocrinol 174:69-75
24. Enhorning S, Wang TJ, Nilsson PM et al (2010) Plasma copeptin and the risk of diabetes mellitus. Circulation 121:2102-2108

25. Lindholm E, Agardh E, Tuomi T, Groop L, Agardh CD (2001) Classifying diabetes according to the new WHO clinical stages. Eur J Epidemiol 17:983-989

26. Cederholm J, Eeg-Olofsson K, Eliasson B et al (2008) Risk prediction of cardiovascular disease in type 2 diabetes: a risk equation from the Swedish National Diabetes Register. Diabetes Care 31: 2038-2043

27. Ludvigsson JF, Andersson E, Ekbom A et al (2011) External review and validation of the Swedish National Inpatient Register. BMC Public Health 11:450

28. Centre for Epidemiology, National Board of Health and Welfare (2003) A finger on the pulse: monitoring public health and social conditions in Sweden 1992-2002. National Board of Health and Welfare, Stockholm

29. Wettermark B, Hammar N, Fored CM et al (2007) The new Swedish Prescribed Drug Register-opportunities for pharmacoepidemiological research and experience from the first six months. Pharmacoepidemiol Drug Saf 16:726-735

30. Jujic A, Nilsson PM, Persson M et al (2016) Atrial natriuretic peptide in the high normal range is associated with lower prevalence of insulin resistance. J Clin Endocrinol Metab 101:1372-1380

31. Magnusson M, Wang TJ, Clish C et al (2015) Dimethylglycine deficiency and the development of diabetes. Diabetes 64:3010 3016

32. Lagerqvist B, James SK, Stenestrand U et al (2007) Long-term outcomes with drug-eluting stents versus bare-metal stents in Sweden. N Engl J Med 356:1009-1019

33. National Board of Health and Welfare (2000) Evaluation of diagnostic quality for acute myocardial infarction in the Swedish Inpatient Registry 1987 and 1995 [in Swedish]. Epidemiologiskt Centrum, Socialstyrelsen, Stockholm

34. Statistics Sweden (2007) MIS 1982:4. Socio-economic classification. In: X11 - Reports on Statistical Co-ordination for the Official Statistics of Sweden. Available from www.scb.se/en /Findingstatistics/Publishing-calendar/Show-detailed-information/? publobjid=6607. Accessed 16 April 2015)

35. Enhorning S, Struck J, Wirfalt E, Hedblad B, Morgenthaler NG, Melander O (2011) Plasma copeptin, a unifying factor behind the metabolic syndrome. J Clin Endocrinol Metab 96:E1065-E1072

36. Kautzky-Willer A, Harreiter J, Pacini G (2016) Sex and gender differences in risk, pathophysiology and complications of type 2 diabetes mellitus. Endocr Rev 37:278-316 